\title{
Changes of spin axis and rate of the asteroid (99942) Apophis during the 2029 close encounter with Earth: A constrained model
}

\author{
J. Souchay ${ }^{1}$, C. Lhotka ${ }^{2}$, G. Heron ${ }^{1}$, Y. Hervé ${ }^{3}$, V. Puente ${ }^{4}$, and M. Folgueira Lopez ${ }^{4}$ \\ ${ }^{1}$ SYRTE, Observatoire de Paris, PSL Research University, CNRS, Sorbonne Université, UPMC Univ. Paris 06, LNE, 61 avenue de \\ l'Observatoire, 75014 Paris, France \\ e-mail: jean.souchay@obspm.fr \\ 2 Space Research Institute, Austrian Academy of Sciences, Schmiedlstrasse 6, 8042 Graz, Austria \\ 3 LATMOS/IPSL, UVSQ Université Paris-Saclay, UPMC Univ. Paris 06, CNRS, Guyancourt, France \\ ${ }^{4}$ Sección Departamental de Astronomía y Geodesia, Facultad de Ciencas Matemáticas, Universidad Complutense Madrid, \\ 28040, Spain
}

Received 27 February 2018 / Accepted 13 May 2018

\begin{abstract}
Context. The dramatic event of the 2029 close encounter between the Earth and the asteroid Apophis on April 132029 at a minimum distance of $38400 \mathrm{~km}$ constitutes an opportunity to make investigations about the modeling of the rotational changes of the asteroid during the event.

Aims. In this paper we deepen a previous study of the rotational changes of Apophis caused by gravitational effects during the close encounter, using important constraints on initial conditions brought by new recent observational data. We also evaluate the effects of the triaxial form of the asteroid on the motion of its axis of rotation in space, as well as the modifications of the spin rate due to tidal deformation.

Methods. First we used more drastic constraints for the physical and geometrical parameters of Apophis, deduced from recent observational campaigns. Second we evaluated the disturbing potential due to the Earth depending not only on the asteroid flattening, but also on the component due to its triaxiality, to deduce the equations of motion and investigate the displacement of the spin axis. Third we estimated what should be the zonal deformation of the asteroid due to the tide exerted by the Earth during the close encounter and we measured the consequences on variations of the spin rate.

Results. We show that the variations of obliquity and precession in longitude of Apophis during the 2029 close encounter can reach very large values, at the level of of respectiveley $1-10^{\circ}$ and $10-40^{\circ}$ depending on geometrical parameters. On the contrary, effects on the spin rate should be relatively small, leading to variations of the sidereal angle of rotation not exceeding $30 \mathrm{~s}$.

Conclusions. The tribute of this paper is to give a constrained model of the important rotational variations of Apophis during its 2029 close encounter with the Earth.
\end{abstract}

Key words. minor planets, asteroids: individual: Apophis - methods: analytical - celestial mechanics

\section{Introduction}

The near Earth asteroid (NEA) 99942 Apophis was discovered at Kitt Peak observatory a little more than one decade ago, on June 19, 2004 (Garradd 2004; Tucker et al. 2004; Smalley 2004). For the circumstances of this discovery we refer the reader to a detailed report by Giorgini et al. (2008). The asteroid will pass at a distance of $38400 \mathrm{~km}$, roughly six radii, from Earth's centre, on April 13, 2029. Although first calculations showed that Apophis could be a potential impactor on our planet during this event, the catastrophic hypothesis was gradually, then definitely rejected with more and more constrained and refined calculations (Sansaturio \& Arratia 2008). Up-to-date ephemerides as HORIZONS from JPL or those devoted to small bodies of the solar system from IMCCE enable to determine the rectangular coordinates of the asteroid before and after the close encounter with an agreement at the level of a relative $10^{-6}$. By using these ephemerides, Souchay et al. (2014a) was able to calculate the orbital parameters of Apophis before and after the 2029 close encounter, quoted as CE in the following. The values, presented in Table 1, show the dramatic changes undergone by these parameters due to the gravitational perturbation of our planet during the $\mathrm{CE}$. We note that some other close encounters are forecast on 2068, 2085 and 2088, with risks of collision which can be considered as negligible. Nevertheless the estimation of these risks seems to be strongly affected by the Yarkovsky effect (Bottke et al. 2006) through its influence on post-2029 orbital predictions (Giorgini et al. 2008; Chesley et al. 2009). Models are refined with the input of new observational data (Bancelin et al. 2012).

In addition to large orbital changes during the $\mathrm{CE}$, Apophis should also undergo significant modifications of its rotational state, as already demonstrated by Scheeres et al. (2005) and Souchay et al. (2014a,b). As in the case of any celestial body this rotational state can be defined by three main components: the first is the locus of the axis of rotation (or of figure) in space; the second is the angular rotational velocity of the asteroid around its rotational axis. A third component is characterized by the motion of the instantaneous axis of rotation with respect to a body-fixed frame. The first component is classically called the combined precession-nutation motion, whereas the second one is directly associated, with the help of a simple integration, to the sidereal angle of rotation. The third can be called "polhody" by referring to the terminology used for the Earth. 
Table 1. Orbital parameters of the asteroid (99942) Apophis before and after the 2029 close encounter with the Earth, taken from Souchay et al. $(2014 a, b)$

\begin{tabular}{lll}
\hline \hline Parameter & Before & After \\
\hline$e$ & 0.191116 & 0.188586 \\
$a[\mathrm{au}]$ & 0.922432 & 1.101692 \\
$i\left[^{\circ}\right]$ & 3.341355 & 2.234257 \\
$q=a(1-e)[\mathrm{au}]$ & 0.746140 & 0.893920 \\
$Q=a(1+e)[\mathrm{au}]$ & 1.098724 & 1.309456 \\
$\Omega\left[^{\circ}\right]$ & 204.61 & 204.29 \\
$\omega\left[^{\circ}\right]$ & 126.54 & 71.88 \\
$T$ [day $]$ & 323.55 & 422.31 \\
\hline
\end{tabular}

Scheeres et al. (2005) showed that terrestrial torques during the flyby should alter the spin state in a dramatic manner that could be observable using ground based telescopes. For the purpose they carried out a Monte Carlo simulation involving flyby geometry in the body fixed space, the moments of inertia and the initial rotation state of the asteroid. Their results were essentially qualitative, with unknown fundamental parameters as the value of the moments of inertia, and the initial nature of the rotational status (short or long axis mode). Nevertheless, their results clearly show that the probability that the rotation be and remains in a short axis mode during the $\mathrm{CE}$ is very high. We note that their study concerns the second and third components of the rotation mentioned above.

In complement, Souchay et al. (2014a,b) studied the first component, in other words, the variations of the position of Apophis angular momentum axis in space during the CE. For this purpose the model is deterministic, based on a Hamiltonian theory for a solid rotational body (Kinoshita 1977) previously applied with good precision for terrestrial planets (Souchay et al. 1999; Bouquillon \& Souchay 1999; Cottereau et al. 2010) as well as for asteroids (Lhotka et al. 2013; Petit et al. 2014). In these studies the equations of motion are given in a rather straightforward manner, depending on a set of a few fundamental parameters, defined in detail in Sect. 3. Among these parameters are the initial value of the obliquity of the asteroid and the values of its moments of inertia, which were still unknown when the study was published.

This paper is highly motivated by new knowledge of the parameters above, due to recent investigations (Pravec et al. 2014) starting from a large set of Apophis light curves obtained by dedicated observational campaigns. In Sect. 2 we make a review of all the new improvements brought by this last paper concerning the rotational characteristics of Apophis that will be fully exploited in the following. Section 3 is devoted to the explanation of the parametrization adequate for our theoretical developments. In Sect. 4 we give the developments of the gravitational potential exerted by the Earth on Apophis during the CE, by taking into account not only the flattening of the asteroid but also its triaxial coefficient which was ignored, meaning it was set to zero in the previous works (Souchay et al. 2014a,b). From that we can deduce the equations of motion of Apophis angular momentum axis in space. Then we integrate these equations numerically to measure the amplitude of this last motion during the close encouter. As a complementary study, we evaluate in Sect. 5 what should be the variations of the spin rate during the CE due to the tidal deformation caused by the Earth gravitational potential.

\section{New constraints on rotation of Apophis coming from Pravec et al. (2014)}

Previous studies concerning the rotational changes of Apophis during the CE (Scheeres et al. 2005; Souchay et al. 2014a,b) were done without any physical and geometrical constraint. In particular fundamental parameters for the calculations as the values of the moments of inertia and the positioning of the spin axis at some given date were not available. The situation has drastically changed since a recent study by Pravec et al. (2014), resulting from a campaign of observations carried out between December 2012 and April 2013. For instance the authors showed that Apophis rotation is retrograde, and that should have interesting implications for impact predictions. For instance Farnochhia et al. (2013) determined a detailed distribution of the post 2029 impacts keyholes to derive probability for several future impact events. For such a study thermal effects depending on the rotational regime are dominant.

First, Pravec et al. (2014) reveal that Apophis is apparently in a state of non-principal axis rotation, that is, in a tumbling state, but still relatively close to the basic rotation state. Indeed the rotational kinetic energy is greater than the minimum kinetic energy by only $2-3 \%$. As a consequence, from the rotation light-curves the authors deduced that the asteroid is in a moderately excited short axis mode (SAM). They gave an accurate estimation of both the rotation period with their corresponding $3 \sigma$ uncertainties: $P_{\phi}=27.38 \pm 0.07 \mathrm{~h}$ and the precession period $P_{\psi}=263 \pm 6 \mathrm{~h}$. This combination of motions leads to the existence of a secondary peak in the light curves with period $P_{1}=30.56 \pm 0.01 \mathrm{~h}$.

Second, one of the important by-products coming from the study above are the very precise determination of the moments of inertia of Apophis, with the following ratios accompanied with their $3 \sigma$ uncertainties: $A / C=0.61_{-0.08}^{+0.11}$ and $B / C=0.965_{-0.015}^{+0.109}$. Therefore we can remark the astonishing fact that the greatest and intermediate moments of inertia differ by only $3-4 \%$.

Third, a fundamental parameter for our study was determined by the authors, that is the direction of the angular momentum vector $\boldsymbol{L}$ in ecliptic coordinates $\lambda_{\mathrm{L}}$ and $\beta_{\mathrm{L}}$. According to Pravec et al. (2014) the nominal values for these coordinates are respectively $\lambda_{\mathrm{L}}=250^{\circ}$ and $\beta_{\mathrm{L}}=-75^{\circ}$ The uncertainty area for $\boldsymbol{L}$ is approximatively an ellipse with the major and minor semiaxes of $27^{\circ}$ and $14^{\circ}$ respectively. More rigorously it can be delimited by a polygone close both to the south ecliptic pole and to the south pole of the current asteroid's heliocentric orbit. We use the values of the pairs $\left(\lambda_{\mathrm{L}}, \beta_{\mathrm{L}}\right)$ corresponding to this polygonal area for our calculations in the following sections. 


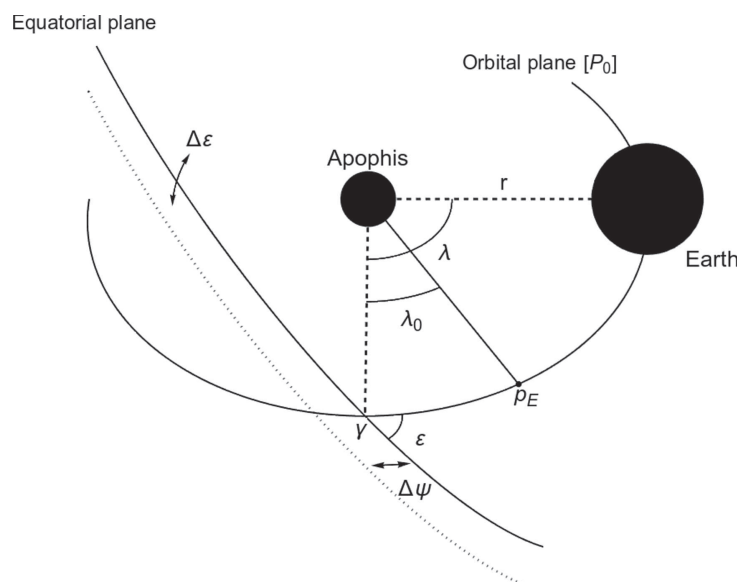

Fig. 1. Parametrization of the Apophis-Earth system during the CE.

\section{Geometrical representation of the orbital and rotational motion of Apophis during the close encounter}

The calculations of the perturbing rotational potential exerted by the Earth on Apophis during the CE necessitates the adoption of a conventional inertial reference plane. As already explained in similar rotational studies, for instance Lhotka et al. (2013) for an application to a set of 100 asteroids, it is recommended to take this reference plane coinciding with the orbital plane of relative motion of the perturbing body considered, with respect to the asteroid centre of mass. This considerably simplifies the calculation of the perturbing gravitational potential. In particular the latitude $\beta$ of the perturbing body is equal to zero. For the study above, by far the predominant perturbing body was the Sun. Therefore the reference plane was the orbital plane of the asteroid considered around the Sun. In the case we consider here the close encounter of the asteroid with the Earth the reference plane is the relative orbital plane of the Earth with respect to Apophis centre of mass.

\subsection{Parametrization of the problem}

Thus, let us call $\left[P_{0}\right]$ the osculating relative orbital plane of the Earth with respect to the Apophis centre of mass during the CE. Here the obliquity $\varepsilon$ of Apophis is determined with respect to $\left[P_{0}\right]$ (Fig. 1) instead of the asteroid orbital plane around the Sun.

We note that the rotation of Apophis is retrograde, as well as the relative orbital motion of the Earth related to the asteroid centre of mass. We have reversed the geometry (Fig. 1) in such a way that the two motions are prograde, thus facilitating the representation of the problem and avoiding sign errors in the analytical expressions presented in the next sections.

Moreover from $\left[P_{0}\right]$ two parameters are sufficient to represent the relative motion of our planet with respect to the centre of mass of the asteroid (Fig. 1): these parameters are $r$, the distance between the centres of mass, and $\tilde{\lambda}$, which is the longitude angle of the Earth centre of mass with respect to a fixed direction which is taken as coincident with the direction of the Earth (namely $p_{\mathrm{E}}$ ) at its minimum distance $(\tilde{\lambda}=0$ at this minimum). Then we define $\gamma$ as the ascending node of the Earth orbital plane with respect to the equator of Apophis and $\lambda_{0}$ the angle between $\gamma$ and $p_{\mathrm{E}}$. To calculate the Earth disturbing potential in the following section we use as fundamental parameter the longitude of the Earth $\lambda$ calculated from $\gamma$, that is to say $: \lambda=\tilde{\lambda}+\lambda_{0}$.

\subsection{Uncertainty of parameters and initial conditions}

The accuracy of our calculations concerning Apophis' rotational changes during the CE is directly dependent on the knowledge of the initial values of the parameters $\varepsilon$ and $\lambda_{0}$, as well as on the accuracy of determination of the two time-dependent variables $\lambda$ and $r$, which characterize the relative orbital motion of the Earth. Concerning these two last variables, Souchay et al. (2014b) have shown that the differences of position given by up-to-date ephemerides as HORIZONS from JPL and the IMCCE portal, which only show up at the seventh or eight digit after the comma are completely negligible. On the other hand, the initial values of the obliquity $\varepsilon_{0}$ and of $\lambda_{0}=\left(\gamma, p_{\mathrm{E}}\right)$ before the $\mathrm{CE}$ are calculated starting from the orientation of the angular momentum vector $\boldsymbol{L}$ which is subject to the uncertainty already detailed at the end of Sect. 2 .

The values of $\left(\lambda_{0}, \varepsilon_{0}\right)$ before the $\mathrm{CE}$ are directly dependent, from a straightforward geometric transformation, on the initial ecliptic coordinates $\left(\lambda_{\mathrm{L}}, \beta_{\mathrm{L}}\right)$ of the polar axis of the asteroid. Therefore in order to explore the possible initial values of the pairs of parameters $\left(\lambda_{0}, \varepsilon_{0}\right)$ for our study, we filled up the polygonal area in Fig. 4 of Pravec et al. (2014) with 10.000 randomly chosen points as follows: the majority $(90 \%)$ of the points are normally distributed with mean vector $\left(250^{\circ},-75^{\circ}\right)$ that can be encircled by an ellipse with minor and major semi-axes, $27^{\circ}$ and $14^{\circ}$, respectively. The remaining $10 \%$ of the points are randomly chosen to fill the complete polygonal region of Fig. 4 of Pravec et al. (2014). The distribution of points $\lambda_{\mathrm{L}}$ and $\beta_{\mathrm{L}}$ are shown in Fig. 2. As we can see, the two distributions peak close (within $1^{\circ}$ ) to the nominal values derived in Pravec et al. (2014) as indicated by the dotted lines.

Then for each pair $\left(\lambda_{\mathrm{L}}, \beta_{\mathrm{L}}\right)$ of random points we calculated $\left(\lambda_{0}, \varepsilon_{0}\right)$ as follows: let $\boldsymbol{r}_{t}=\boldsymbol{r}_{\mathrm{E}}(t)-\boldsymbol{r}_{\mathrm{A}}(t)$ be the position of the Earth relative to Apophis at instant $t$. We normalize $r_{t}$ to be of unit length. The instantaneous orbit normal $\boldsymbol{o}_{\mathrm{t}}$ of the orbital plane of the Earth relative to Apophis is then given by $\boldsymbol{o}_{\mathrm{t}}=\boldsymbol{r}_{t} \times \boldsymbol{r}_{t+1}$. From $\boldsymbol{f}=(\cos \beta \cos \lambda, \cos \beta \sin \lambda, \sin \beta)$ we then have $\cos \varepsilon_{t}=\boldsymbol{f} \cdot \boldsymbol{o}_{\mathrm{t}}$. Next, 

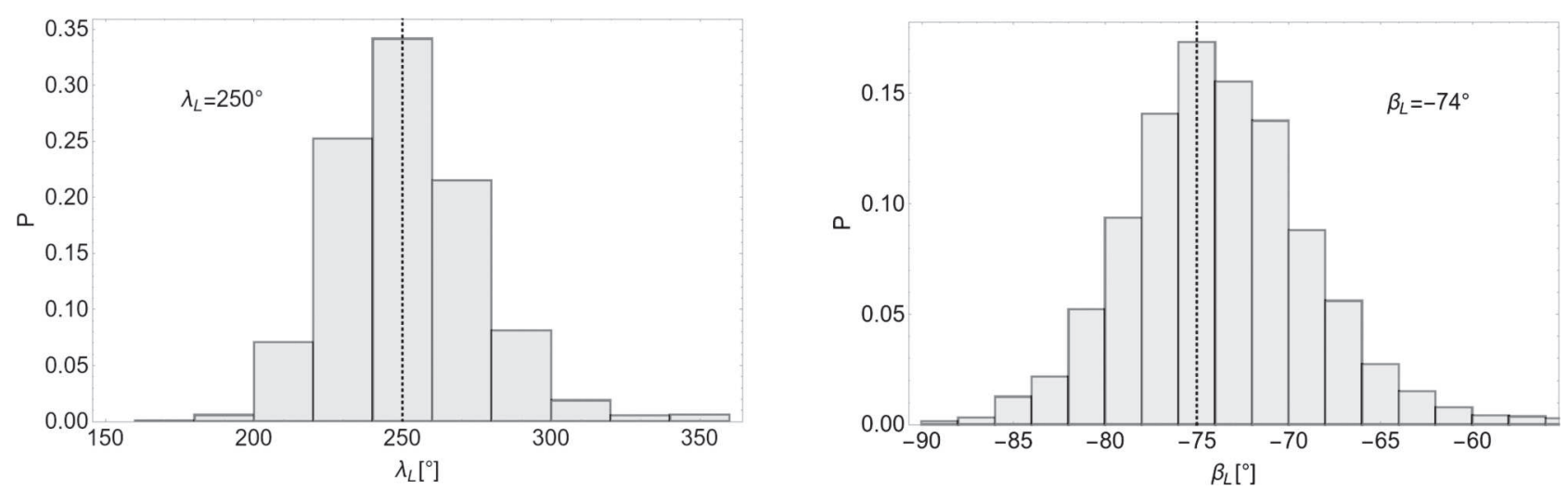

Fig. 2. Distribution of the values of the ecliptic longitude $\lambda_{\mathrm{L}}$ (left panel) and latitude $\beta_{\mathrm{L}}$ (right panel) of Apophis polar axis from our set of 10000 points in the polygonal zone delimited by Pravec et al. (2014). The dotted line corresponds to the nominal value in this paper, mentioned in the figure. The grey bin corresponds to the peak of the distribution.

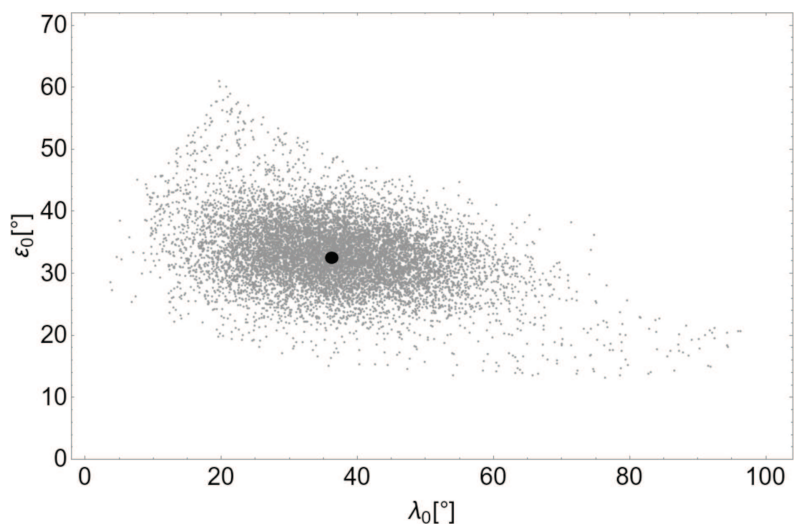

Fig. 3. Solutions in the $\left(\lambda_{0}, \varepsilon_{0}\right)$-plane, from the set of 10000 initial conditions for $\left(\lambda_{\mathrm{L}}, \beta_{\mathrm{L}}\right)$.
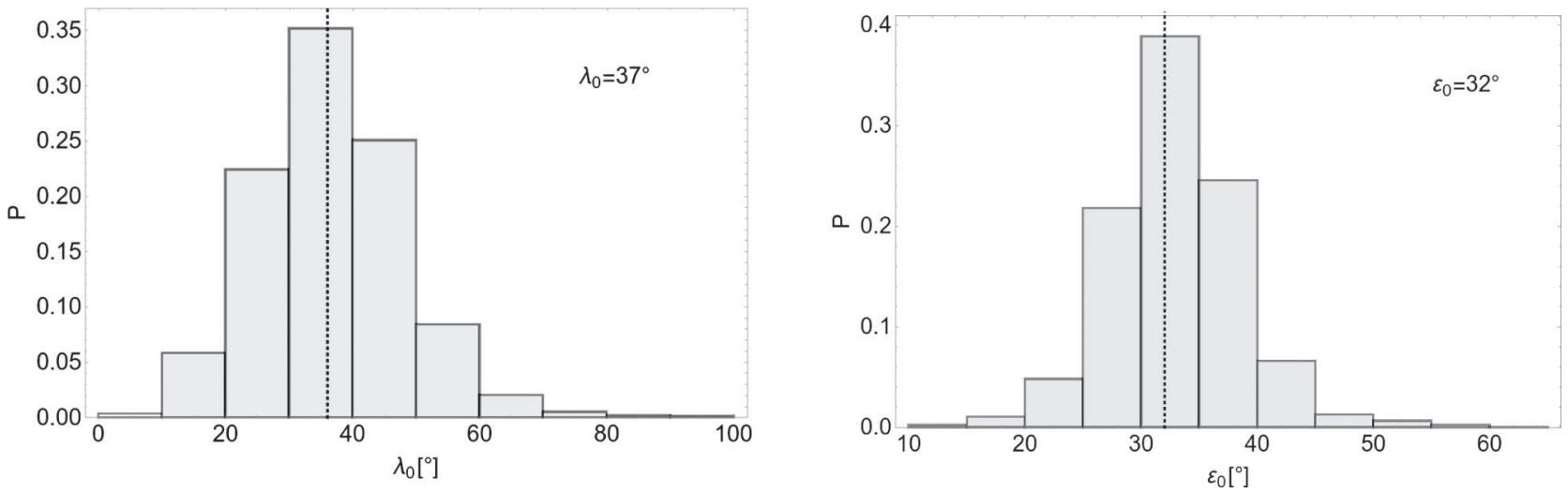

Fig. 4. Distribution of $\lambda_{0}$ (left panel) and $\varepsilon_{0}$ (right panel) from the set of 10000 initial conditions for $\left(\lambda_{\mathrm{L}}, \beta_{\mathrm{L}}\right.$ ). The dotted line corresponds to the nominal value in this paper, mentioned in the figure. The grey bin corresponds to the peak of the distribution.

we calculate $\Gamma_{\mathrm{t}}=\boldsymbol{f} \times \boldsymbol{o}_{\mathrm{t}} / \sin \varepsilon_{\mathrm{t}}$ and find $\cos \lambda_{\mathrm{t}}=\Gamma_{\mathrm{t}} \cdot \boldsymbol{o}_{\mathrm{t}}$. At time of closest approach, say $t=0$, we find the pair $\left(\lambda_{0}, \varepsilon_{0}\right)$ for each pair of $(\lambda, \beta)$. The results are shown in Fig. 3, the nominal solution being indicated by the black dot.

Finally, we show the distribution of possible values of $\varepsilon_{0}$ and of $\lambda_{0}$ in Fig. 4 . They peak at the values $\lambda_{0} \simeq 37^{\circ}$ and $\varepsilon_{0} \simeq 32^{\circ}$ that also correspond to the values directly obtained from the nominal values $\lambda_{\mathrm{L}}=250^{\circ}, \beta_{\mathrm{L}}=-75^{\circ}$ of Pravec et al. (2014).

\section{Variations of Apophis spin axis during the close encounter}

To calculate the variations of the orientation of Apophis angular momentum during the CE, we were able to use the classical theory of Kinoshita (1977) for the rotation of a rigid body (here Apophis) undergoing the gravitational potential exerted by an external body (here the Earth). 


\subsection{Theoretical expressions for Apophis rotational potential}

This rotational potential can be expressed as follows:

$U=U_{1}+U_{2}$

where we split the gravitational potential into two parts:

$U_{1}=\frac{G M_{\oplus}}{r^{3}}\left[\frac{2 C-A-B}{2} P_{2}(\sin \delta)+\frac{A-B}{4} P_{2}^{2}(\sin \delta) \cos 2 \alpha\right]$

and into the part that includes the higher order harmonics:

$U_{2}=\sum_{n=3}^{\infty} \frac{G M M_{\oplus} a_{\mathrm{A}}^{n}}{r^{n+1}}\left[J_{n} P_{n}(\sin \delta)-\sum_{m=1}^{n} P_{n}^{m}(\sin \delta) \times\left(C_{\mathrm{nm}} \cos m \alpha+S_{\mathrm{nm}} \sin m \alpha\right)\right]$

Here, $G$ is the gravitational constant, $M$ stands for the mass of Apophis, $a_{\mathrm{A}}$ its mean radius, and $A, B$ and $C$ are the moments of inertia along the principal axes of the asteroid $(A<B<C)$. Parameter $M_{\oplus}$ is the mass of the Earth, $\alpha$ is the longitude of the perturbing body (the Earth) counted from a conventional zero meridian on the asteroid (in that sense it must not be confused with the classical right ascension) and $\delta$ represents its declination with respect to Apophis equatorial plane. The $P_{i}^{j}$ represent the Legendre polynomials of degree $i$ and order $j$. Here we restrict to the first order part $U_{1}$ of the potential. The Legendre polynomial $P_{2}(\sin \delta$ ) and $P_{2}^{2}(\sin \delta) \cos \alpha$ can be developed in the following form (Kinoshita 1977):

$$
\begin{aligned}
P_{2}(\sin \delta)= & \frac{1}{2}\left(3 \cos ^{2} J-1\right)\left[\frac{1}{2}\left(3 \cos ^{2} I-1\right) P_{2}(\sin \beta)-\frac{1}{2} \sin 2 I P_{1}^{2} \sin (\beta) \times \sin (\lambda-h)-\frac{1}{4} \sin ^{2} I P_{2}^{2}(\sin \beta) \cos (2 \lambda-h)\right] \\
+ & \sin 2 J\left[-\frac{3}{4} \sin 2 I P_{2} \sin (\beta) \cos g-\sum_{\epsilon= \pm 1} \frac{1}{4}(1+\epsilon \cos I) \times(-1+2 \epsilon \cos I) P_{2}^{1}(\sin \beta) \sin (\lambda-h-\epsilon g)\right. \\
& \left.-\sum_{\epsilon= \pm 1} \frac{1}{8} \epsilon \sin I(1+\epsilon \cos I) P_{2}^{2} \sin (\beta) \cos (2 \lambda-2 h-\epsilon g)\right] \\
+\sin ^{2} J\left[\frac{3}{4} \sin ^{2} I P_{2}(\sin \beta) \cos 2 g+\frac{1}{4} \sum_{\epsilon= \pm 1} \epsilon \sin I \times(1+\epsilon \cos I) P_{2}^{1} \sin (\beta) \sin (\lambda-h-2 \epsilon g)-\frac{1}{16}\right. & \\
& \left.\times \sum_{\epsilon= \pm 1}(1+\epsilon \cos I)^{2} P_{2}^{2}(\sin \beta) \cos 2(\lambda-h-\epsilon g)\right]
\end{aligned}
$$

and

$P_{2}^{2}(\sin \delta) \cos 2 \alpha=3 \sin ^{2} J\left[-\frac{1}{2}\left(3 \cos ^{2} I-1\right) P_{2}(\sin \beta) \cos 2 l+\frac{1}{2} \sum_{\epsilon= \pm 1} \sin 2 I P_{2}^{1}(\sin \beta) \sin (\lambda-h-2 \epsilon l)\right.$

$$
\begin{aligned}
& \left.+\frac{1}{8} \sin ^{2} I P_{2}^{2}(\sin \beta) \cos (\lambda-h-2 \epsilon l)\right] \\
& +\sum_{\rho= \pm 1} \rho \sin J(1+\rho \cos J)\left[-\frac{3}{2} \sin 2 I P_{2}(\sin \beta) \cos (2 \rho l+g)\right. \\
& +\sum_{\epsilon= \pm 1} \frac{1}{2}(1+\epsilon \cos I) \times(-1+2 \epsilon \cos I) P_{2}^{1}(\sin \beta) \sin (\lambda-h-2 \rho \epsilon l-\epsilon g) \\
& \left.+\sum_{\epsilon= \pm 1} \frac{1}{4} \epsilon \sin I(1+\epsilon \cos I) P_{2}^{2}(\sin \beta) \times \cos (2 \lambda-2 h-2 \rho \epsilon l-\epsilon g)\right] \\
& +\sum_{\rho= \pm 1} \frac{1}{4}(1+\rho \cos J)^{2}\left[-3 \sin ^{2} I P_{2}^{1}(\sin \beta) \cos (2 l+2 \rho g)-\sum_{\epsilon= \pm 1} \epsilon \sin I(1+\epsilon \cos I) P_{2}^{1}(\sin \beta)\right. \\
& \left.\quad \times \sin (\lambda-h-2 \rho \epsilon l-2 \epsilon g)+\sum_{\epsilon= \pm 1} \frac{1}{4}(1+\epsilon \cos I)^{2} \times P_{2}^{2}(\sin \beta) \cos 2(\lambda-h-\rho \epsilon l-\epsilon g)\right]
\end{aligned}
$$

For the definition of the parameters involved, we can refer to this last paper. $l, g$ and $h$ are the Andoyer rotational angles, whereas $I$ is the obliquity angle $(I=-\varepsilon)$ between the reference plane $\left[P_{0}\right]$ and the plane perpendicular to the angular momentum vector (see Fig. 1). $J$ represents the angle between the angular momentum axis and the figure axis. The longitude $\lambda$ has been defined in the precedent section, whereas $\beta$ designates the latitude of the perturbing body (the Earth) with respect to $\left[P_{0}\right]$. As a consequence of our choice for this last plane, we have: $\beta=0$. The sidereal rotation of Apophis is given by the combination :

$\Phi=l+g=\omega t+\Phi_{0}$

where $\omega=2 \pi / T, T$ being the period of rotation of the asteroid, and $\Phi_{0}$ is an initial phase at $t=0$. The two main parameters investigated here are those representing the motion of the Apophis axis in space, that is to say $\psi=-h$ and $\varepsilon=-I$ 


\subsection{Simplified expression for the potential}

In fact the analytical developments (4) and (5) can be considerably simplified with the substitution $\beta=0$ and the assumption that $J \approx 0$ which means that the axis of angular momentum, the axis of figure and also the axis of rotation of the asteroid are quasi coincident. As explained previously the probability that such a condition is fulfilled is quite high, as given the fact that, according to Pravec et al. (2014), the rotational regime is close to a short axis mode (SAM), with a ratio of the rotational kinetic energy to the basic spin state energy $E / E_{0}=1.224 \pm 0.013$ Then the axis of figure and the axis of rotation should be nearly coinciding . Thus the rotational potential at first order $U_{1}$ given by (2) can be reduced to the two shortened components below:

$U_{1}=U_{1, a}+U_{1, b}$

$U_{1, a}$ and $U_{1, b}$ depending directly respectively of the flattening and triaxiality:

$U_{1, a}=\frac{\mathrm{G} M_{\oplus}}{r^{3}} \frac{2 C-A-B}{2} \times\left(-\frac{1}{4}\left(3 \cos ^{2} I-1\right)-\frac{3}{4} \sin ^{2} I \cos 2(\lambda-h)\right)$

and

$U_{1, b}=\frac{G M_{\oplus}}{r^{3}} \frac{A-B}{4} \times\left(\frac{3}{2} \sin ^{2} I \cos 2(l+g)+\frac{3}{4}(1+\cos I)^{2} \cos 2(\lambda-h-l-g)+\frac{3}{4}(1-\cos I)^{2} \cos 2(\lambda-h+l+g)\right)$

\subsection{Equations for the precession and obliquity}

Following Kinoshita's theory and using the same kind of developments as preliminary ones by Souchay et al. (2014a,b) we give the bi-dimensional variations of Apophis axis in space during the CE:

$[\Delta \psi]_{\mathrm{CE}}=-\Delta h=\frac{1}{G \sin I} \frac{\partial W}{\partial I}$

$[\Delta \varepsilon]_{\mathrm{CE}}=-\Delta I=-\frac{1}{G \sin I} \frac{\partial W}{\partial h}+\frac{\cot I}{G} \frac{\partial W}{\partial g}$

where $G$, the angular momentum component of the asteroid corresponding the Andoyer action variable given by $G=C \omega$. $W$ is the determining function at first order given by integration of the potential:

$W=\int\left(U_{1, a}+U_{1, b}\right) \mathrm{d} t$

Combining these last equations, we find:

$$
\begin{aligned}
t_{\mathrm{CE}}= & \frac{K}{2} \int \cos I\left(\frac{a}{r}\right)^{3}(1-\cos 2(\lambda-h)) \mathrm{d} t-\frac{K^{\prime}}{2} \int\left(\frac{a}{r}\right)^{3}(2 \cos I \cos 2(l+g)-(1+\cos I) \cos 2(\lambda-h-l-g) \\
& +(1-\cos I) \cos 2(\lambda-h+l+g)) \mathrm{d} t \\
t_{\mathrm{CE}}= & \frac{K}{2} \int \sin I\left(\frac{a}{r}\right)^{3} \sin 2(\lambda-h) \mathrm{d} t+K^{\prime} \int \cos I\left(\frac{a}{r}\right)^{3} \sin I \sin 2(l+g) \mathrm{d} t+\frac{K^{\prime}}{2} \int \frac{1}{\sin I}\left(\frac{a}{r}\right)^{3}\left[(1+\cos I)^{2} \sin 2(\lambda-h-l-g)\right. \\
+ & \left.(1-\cos I)^{2} \sin 2(\lambda-h+l+g)\right] \mathrm{d} t-\frac{K^{\prime}}{2} \int \frac{\cos I}{\sin I}\left(\frac{a}{r}\right)^{3}\left[(1+\cos I)^{2} \sin 2(\lambda-h-l-g)-(1-\cos I)^{2} \sin 2(\lambda-h+l+g)\right] \mathrm{d} t
\end{aligned}
$$

where the scaling constants $K$ and $K^{\prime}$ have the following form:

$K=\frac{3 G M_{\oplus}}{a^{3} \omega} H_{\mathrm{d}}$ and $K^{\prime}=\frac{3 G M_{\oplus}}{a^{3} \omega} H_{\mathrm{t}}$

with

$H_{\mathrm{d}}=\frac{2 C-A-B}{2 C}, H_{t}=\frac{B-A}{4 C}$

where $H_{\mathrm{d}}$ and $H_{\mathrm{t}}$ are the dynamical flattening and triaxiality of Apophis. The choice of $a$ is conventional. Here $a$ is taken as the minimum distance during the $\mathrm{CE}$. 


\subsection{Important effects of the triaxiality}

In general for the large bodies of our solar system and the planets in particular, the triaxiality, characterized by $H_{\mathrm{t}}$ and concerning the terms with $K^{\prime}$ at the right hand side of (13) and (14), affects poorly the motion of the spin axis in space. This is clearly demonstrated in the case of the Earth (Kinoshita 1977; Souchay et al. 1999) or Mars (Bouquillon \& Souchay 1999). Two reasons explain this fact. First the triaxiality coefficient $H_{\mathrm{t}}$ given by (16) is generally very small for these celestial bodies in comparison with the corresponding dynamical ellipticity $H_{\mathrm{d}}$. This is due to their figure close to axi-symmetry. For instance for the Earth we have: $H_{\mathrm{t}} / H_{\mathrm{d}}=0.00163$ (Souchay et al. 1999). Second the expression of the potential term $U_{1, b}$ related to the triaxiality developed at the right hand side of (9) includes the high frequency argument $l+g$ representing the sidereal rotation of the planet, whereas the potential term $U_{1, a}$ related to the dynamical ellipticity at the right-hand side of (8) depends on the low frequency variable $\lambda$. Then, after integration, this considerably lowers the amplitude of the triaxiality terms that are proportional to $H_{\mathrm{t}}$ in comparison to those proportional to $H_{\mathrm{d}}$.

In the case of Apophis, from the values of the ratio $A / C$ and $B / C$ given in Sect. 2 application of (16) leads to : $H_{\mathrm{d}}=0.2125$ and $H_{\mathrm{t}}=0.08875$. That gives $H_{\mathrm{t}} / H_{\mathrm{d}}=0.4164$ which means that the second coefficient is about the same order as the first one. Moreover, both arguments $\lambda$ and $l+g$ have a high frequency and should lead to small amplitudes after integration. But this is greatly compensated by the scaling factors $K$ and $K^{\prime}$ which have a very large amplitude because of the small distance of the Earth during the $\mathrm{CE}$, which corresponds to a small value of $a$ at the denominator of $K$ and $K^{\prime}$ in (16). For comparison, the application of the formula (15) gives $K=355.8^{\circ} / d$ and $K^{\prime}=148.2^{\circ} / d$ which are considerably bigger, by seven orders of magnitude, than the corresponding scaling factors $K_{\text {Moon }}=7553^{\prime \prime} / c y=5.744 \times 10^{-5} \% / d$ and $K_{\text {Sun }}=3483^{\prime \prime} / c y=2.649 \times 10^{-5} \circ / d$ used as scaling factors to calculate the precession and nutation of the Earth due respectively to the Moon and to the Sun (Kinoshita 1977). In other words we can assert that the efficiency of the gravitational effect of the Earth during the small time interval of the CE is $6.194 \times 10^{6}$ and $1.343 \times 10^{7}$ larger than the corresponding effect respectively of the Moon and of the Sun on the combined precession-nutation motion of our planet. Therefore we can expect that in a few hours of close encounter the spin axis of Apophis will change with a larger amplitude than during several thousands years for the Earth, that will be supported by our results in the next section. In the following we carry out numerical simulations to determine what should be the variations $\Delta \psi=-\Delta h$ and $\Delta \varepsilon=-\Delta I$ respectively of the precession in longitude and of the obliquity of the asteroid.

\subsection{Numerical results}

For our numerical simulations we directly integrate Eqs. (13) and (14) starting from each of the 10000 pairs of initial conditions $\left(\lambda_{0}, \varepsilon_{0}\right)$ determined in the previous section. For each pair we investigated the amplitudes of the variations $\Delta \varepsilon$ and $\Delta \psi$ during the CE. We remind the reader that $\Delta \varepsilon$ and $\Delta \psi$ represent respectively the variations of obliquity and of the longitude of the node of the equator of Apophis with respect to the plane of relative motion of the Earth. We observe that these variations are very sensitive to the initial conditions. As shown in Fig. 5 (left panel), the amplitude of $\Delta \psi$ varies between the extrema $7^{\circ}$ and $38^{\circ}$ according those initial conditions. The minimum value is obtained for the pair $\left(\lambda_{0}, \varepsilon_{0}\right)=\left(19.7^{\circ}, 60.9^{\circ}\right)$ and the maximum one for $\left(\lambda_{0}, \varepsilon_{0}\right)=\left(96.4^{\circ}, 20.6^{\circ}\right)$. For the nominal value $\left(\lambda_{0}, \varepsilon_{0}\right)=\left(36^{\circ}, 32^{\circ}\right)$ the variation is $\Delta \psi=20^{\circ}$.

As shown in Fig. 5 (right panel), the amplitudes of the absolute variations of $\Delta \varepsilon$ are globally significantly less important than for $\Delta \psi$, ranging from $1.1^{\circ}$ and $6.1^{\circ}$ obtained respectively for the pairs $\left(\lambda_{0}, \varepsilon_{0}\right)=\left(7.7^{\circ}, 45.1^{\circ}\right)$ and $\left(\lambda_{0}, \varepsilon_{0}\right)=\left(55.0^{\circ}, 44.6^{\circ}\right)$. For the nominal value $\left(\lambda_{0}, \varepsilon_{0}\right)=\left(36^{\circ}, 32^{\circ}\right)$ the variation is $\Delta \varepsilon=3.5^{\circ}$. The sign of the variations is always negative, as can be observed in Fig. 5 (right panel). This means that in any case the close encounter of the asteroid with the Earth is characterized by a diminution of its obliquity which looks like an intuitive fact : the gravitational attraction of the Earth tends to tilt the axis towards an alignment of the equatorial plane with the direction of the Earth.

Figure 6 show respectively the histograms of the absolute variations of the two parameters. We note that $\Delta \psi$ has much more probability $(85 \%)$ to occur between $15^{\circ}$ and $25^{\circ}$ than outside this interval. In parallel $\Delta \varepsilon$ has much more probability $(85 \%)$ to occur between $2.6^{\circ}$ and $5.4^{\circ}$.

As explained in details in Sect. 4.4 the influence of the triaxiality parameter $H_{\mathrm{t}}$ on the values obtained for $\Delta \psi$ and $\Delta \varepsilon$ can be particularly big. From our set of 10000 initial points we have got the maximum differences of $\Delta(\Delta \psi)=15.7^{\circ}$ and $\Delta(\Delta \varepsilon)=4.9^{\circ}$ between the amplitudes when setting the value of the triaxiality coefficient to $H_{\mathrm{t}}=0$ or to its real value $H_{\mathrm{t}}=0.08875$. They correspond respectively to the pairs $\left(\lambda_{0}, \varepsilon_{0}\right)=\left(96.4^{\circ}, 2.6^{\circ}\right)$ and $\left(\lambda_{0}, \varepsilon_{0}\right)=\left(19.7^{\circ}, 60.9^{\circ}\right)$. These differences are plotted respectively in Fig. 7.

Finally from our calculations it is possible to establish a three-dimensional colour diagram representing the variations of $\Delta \psi$ and $\Delta \varepsilon$ as a function of the initial values $\left(\lambda_{0}, \varepsilon_{0}\right)$, respectively in Fig. 8. Concerning $\Delta \psi$ (Fig. 8, left panel) the amplitudes have their largest values for $\lambda_{0}$ close to $90^{\circ}$, and decrease globally with this parameter. Concerning $\Delta \varepsilon$ (Fig. 8 , right panel) the maximum values are found inside a circular zone centred around $\lambda_{0}=60^{\circ}$ and $\varepsilon_{0}=45^{\circ}$.

\section{Variations of Apophis spin rate during the CE}

To complete our study dealing with the rotational changes of Apophis during the CE our purpose in this section is to evaluate the level of variation of Apophis spin rate due to the tidal deformations caused by the Earth approcahing the asteroid as a perturbing body. The underlying concept is the conservation of the angular momentum which can be written at first approximation:

$\mathcal{H}=C \times \omega \frac{\mathrm{d} \omega}{\omega}=-\frac{\mathrm{d} C}{C}$ 

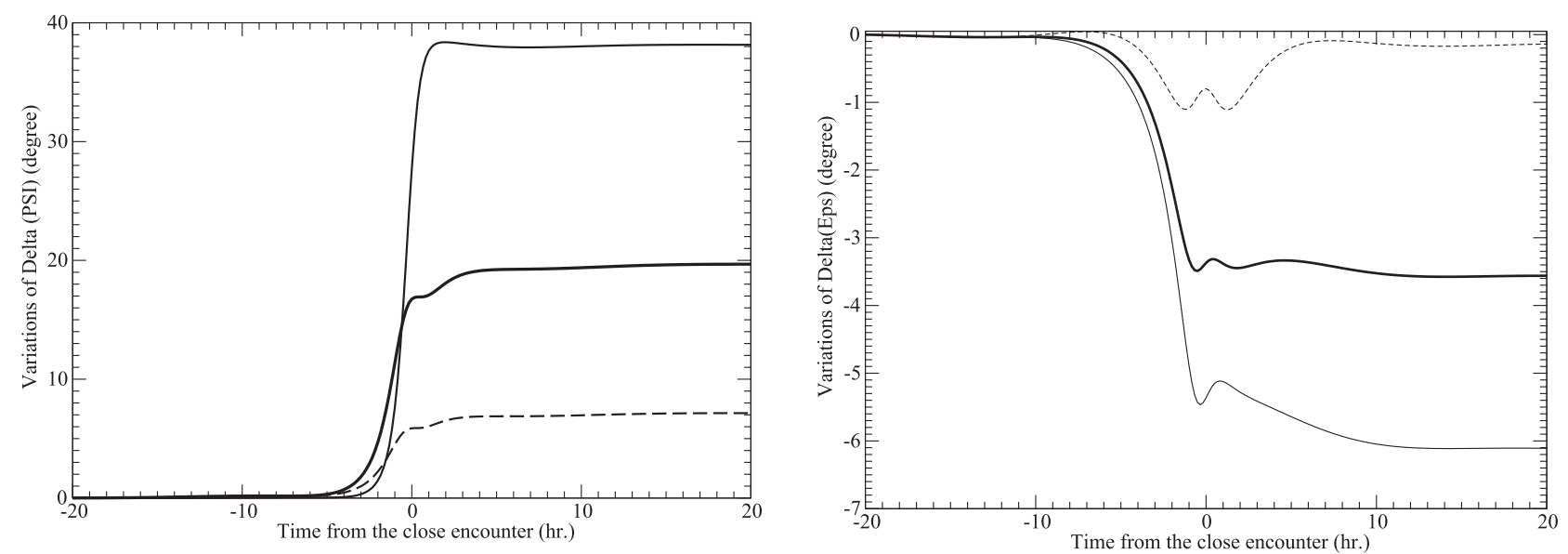

Fig. 5. Maximum (full) and minimum (dashed) variations of $\Delta \psi(l e f t)$ and $\Delta \varepsilon$ (right) during the close encounter between Apophis and the Earth, respectively for the set of pairs $\left(\lambda_{0}, \varepsilon_{0}\right)=\left(96.4^{\circ}, 20.6^{\circ}\right)$ and $\left(\lambda_{0}, \varepsilon_{0}\right)=\left(19.7^{\circ}, 60.9^{\circ}\right)$. In bold the corresponding variations for the nominal values $\left(\lambda_{0}, \varepsilon_{0}\right)=\left(36^{\circ}, 32^{\circ}\right)$.
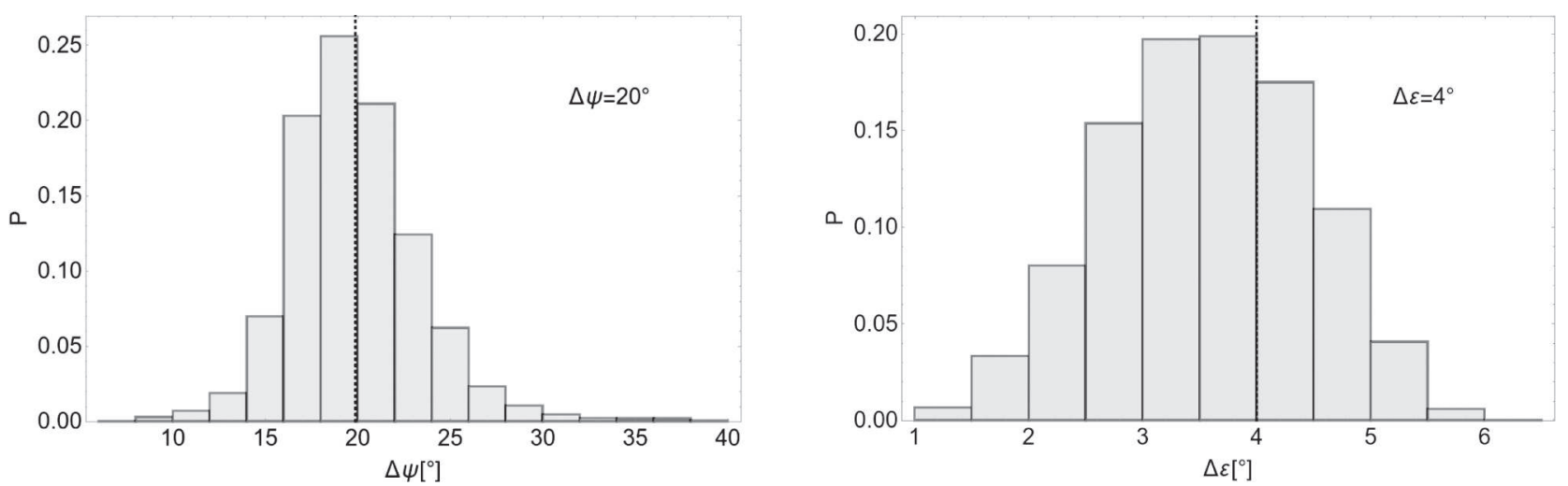

Fig. 6. Histogram of the variations of $\Delta \psi$ (left) and $\Delta \varepsilon$ (right) during the close encounter with a sample of 10000 initial values for $\left(\lambda_{0}, \varepsilon_{0}\right)$. The dotted line corresponds to the nominal value, mentioned in the figure. The grey bin corresponds to the peak of the distribution.
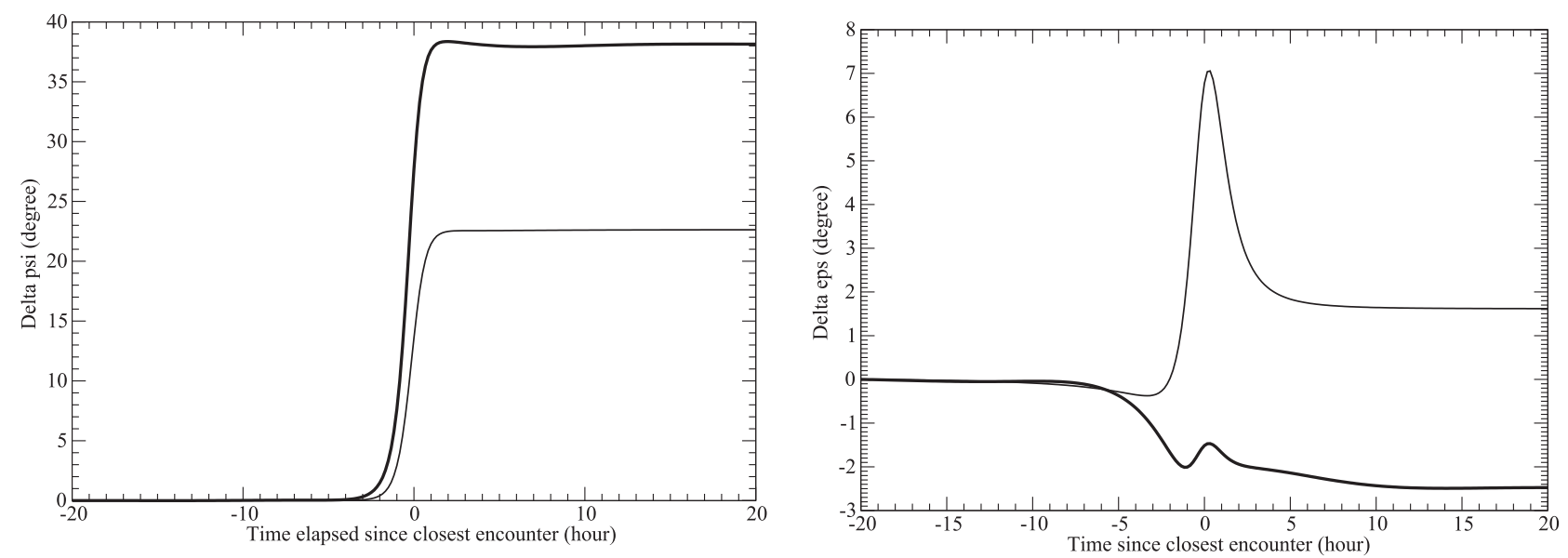

Fig. 7. Variations of $\Delta \psi($ left $)$ and $\Delta \varepsilon$ (right) for $\left(\lambda_{0}, \varepsilon_{0}\right)=\left(96.3^{\circ}, 20.6^{\circ}\right)$ when setting the value of $H_{\mathrm{t}}=0$ (dashed) and $H_{t}=0.08875$ (full).

where $\mathcal{H}$ is the angular momentum, $\omega$ represents the spin frequency $\left(\frac{\omega=2 \pi}{T}\right)$. Here we consider that the asteroid rotates in a short axis mode, which supposes that the axis of figure and the axis of rotation of the asteroid can be considered as coinciding. This reduces to zero the components of $\mathcal{H}$ along the two rectangular axes perpendicular to the polar axis. The contant value of $\mathcal{H}$ during the $\mathrm{CE}$ leads to:

$\frac{\mathrm{d} \omega}{\omega}=-\frac{\mathrm{d} C}{C}$

where $\mathrm{d} C$ an $\mathrm{d} \omega$ represent respectively the variations of $C$ and $\omega$ related to the tidal deformations exerted by the Earth. 

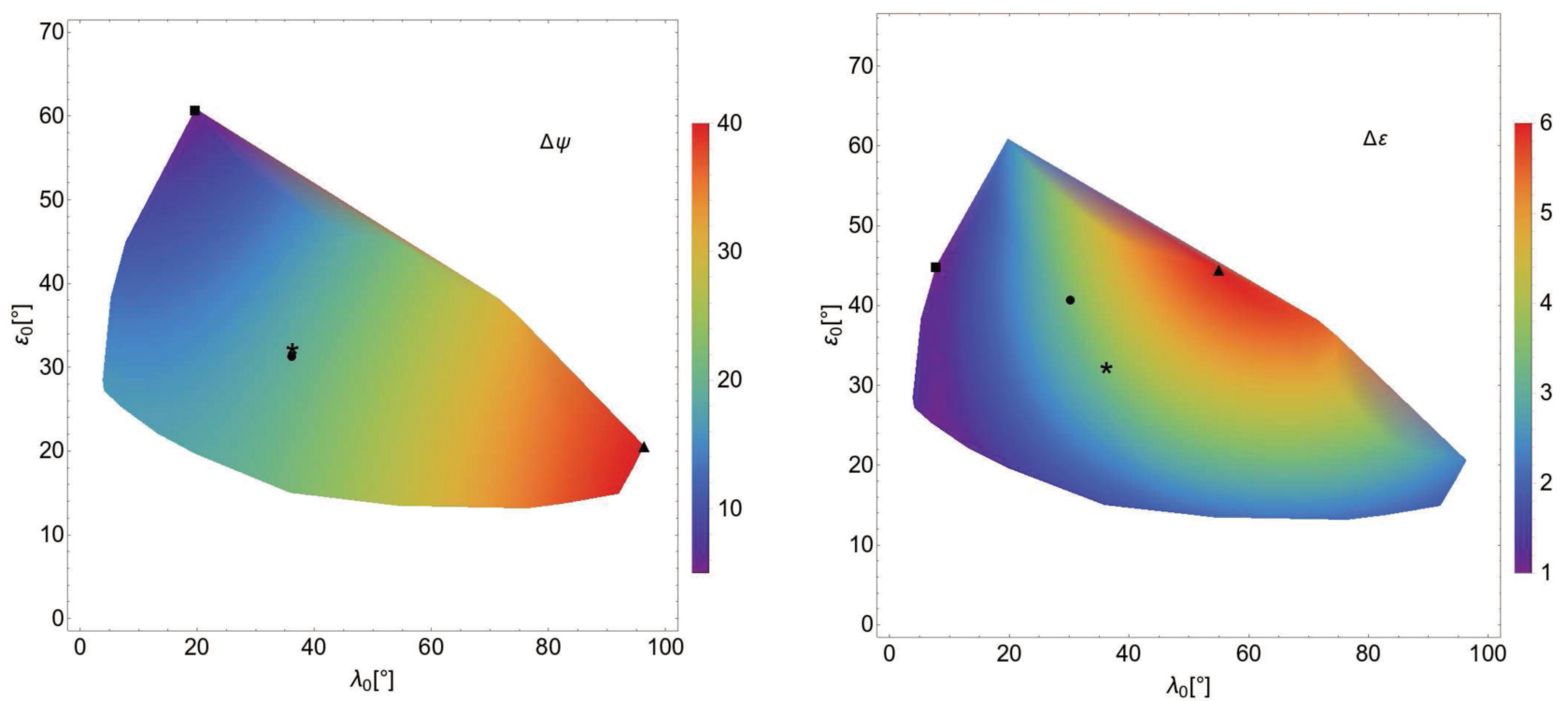

Fig. 8. Variations of $\Delta \psi($ left $)$ and $\Delta \varepsilon$ (right) during the close encounter with a sample of 10000 initial values for $\left(\lambda_{0}, \varepsilon_{0}\right)$. Minimum (square), maximum (triangle), nominal (star) and most common (circle) values. Units at the right hand side are in degrees.

\subsection{Zonal deformations and expression of $\mathrm{d} \omega / \omega$}

To calculate the deformations of Apophis produced by the zonal part of the tidal potential $\mathcal{U}$ due to the Earth and the related amplitude of $\mathrm{d} C$ above we can refer to methods given by classical textbooks as Melchior (1983). Thus the bulge produced by this zonal part at a given point of the asteroid with co-latitude $\theta$ is represented by a differential potential $\Delta \mathcal{U}$ as follows:

$\Delta \mathcal{U}=G \frac{\mathrm{d} C-\mathrm{d} A}{2 a_{\mathrm{A}}^{3}}\left(3 \cos ^{2} \theta-1\right)\left(\frac{a_{\mathrm{A}}}{r}\right)^{3}$

In the present situation, $a_{\mathrm{A}}$ is the mean radius of the asteroid. By following the developments from Melchior and adapting them to the present case we find:

$\frac{3}{4} k_{2} G M_{\oplus}\left(\frac{a_{\mathrm{A}}^{2}}{r^{3}}\right)\left(\sin ^{2} \delta-\frac{1}{3}\right)=\frac{G M_{\mathrm{A}}}{2 a_{\mathrm{A}}} \frac{\mathrm{d} C-\mathrm{d} A}{M_{\mathrm{A}} a_{\mathrm{A}}^{3}}$

where $k_{2}$ and $M_{\mathrm{A}}$ are respectively the Love number and the mass of the asteroid, and $r$ the distance between the asteroid and the Earth barycentres. Here we suppose that the asteroid reacts like a planet, that is to say that it undergoes elastic deformation whose response is characterized by $k_{2}$. As we consider here a purely zonal deformation: $\mathrm{d} A=\mathrm{d} B$. Moreover the central moment of inertia $I$ remains constant during the deformation, $\mathrm{d} I=1 / 2(\mathrm{~d} A+\mathrm{d} B+\mathrm{d} C)=0$, so that finally we have $\mathrm{d} A=-\mathrm{d} C / 2$.

Taking into account this property, we can deduce the expression of $\mathrm{d} C$

$\frac{\mathrm{d} C}{M a_{\mathrm{A}}^{2}}=k_{2} \frac{M_{\oplus}}{M_{\mathrm{a}}}\left(\frac{a_{\mathrm{A}}}{r}\right)^{3}\left(\sin ^{2} \delta-\frac{1}{3}\right)$

With $C \approx 0.334 M a_{\mathrm{A}}^{2}$ and combining this last equation with (17) we finally obtain :

$\frac{\mathrm{d} \omega}{\omega}=-\frac{k_{2}}{0.334} \frac{M_{\oplus}}{M_{\mathrm{a}}}\left(\frac{a_{\mathrm{A}}}{r}\right)^{3}\left(\sin ^{2} \delta-\frac{1}{3}\right)$

This equation can be transformed in such a way that it involves the relative bulk density of the Earth $\left(\rho_{\oplus}\right)$ and of the asteroid $\rho_{\mathrm{A}}$ Indeed we have :

$M_{\mathrm{A}}=\rho_{\mathrm{A}} \times \frac{4}{3} \pi a_{\mathrm{A}}^{3} \quad M_{\oplus}=\rho_{\oplus} \times \frac{4}{3} \pi R_{\oplus}^{3}$

with $R_{\oplus}$ the mean radius of the Earth.

Moreover according to our choice of geometric parametrization (cf. Fig. 1), the declination of the Earth $\delta$ measured with respect to Apophis equatorial plane is given by :

$\sin \delta=\sin I \sin \lambda$

Thus (21) can be finally rewritten as :

$\frac{\mathrm{d} \omega}{\omega}=-\frac{k_{2}}{0.334} \frac{\rho_{\oplus}}{\rho_{\mathrm{A}}}\left(\frac{R_{\oplus}}{r}\right)^{3}\left(\sin ^{2} I \sin ^{2} \lambda-\frac{1}{3}\right)$ 


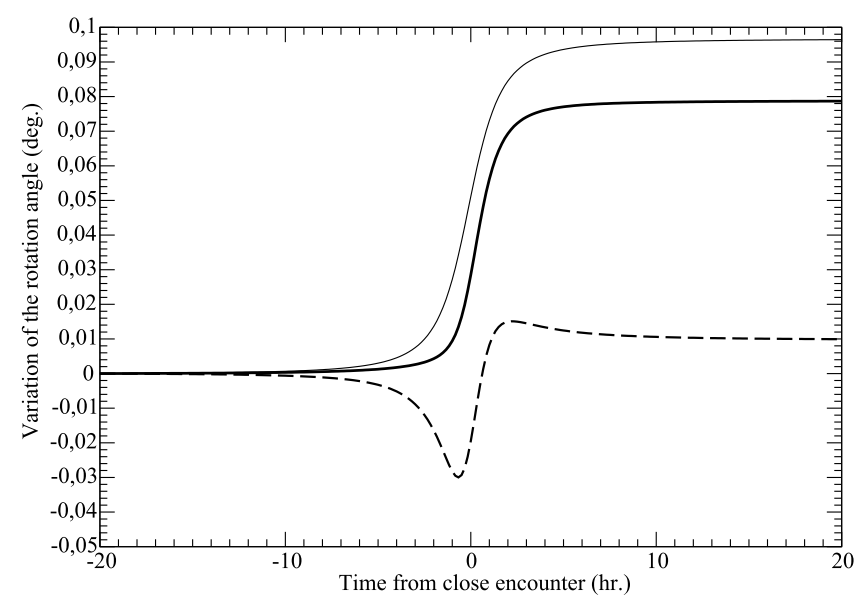

Fig. 9. Variations of the proper rotation angle $\Delta \Phi$ during the close encounter for three different set of initial values as in Fig. 5 , i.e. $\left(\lambda_{0}, \varepsilon_{0}\right)=$ $\left(36^{\circ}, 32^{\circ}\right)$ (bold), $\left(96.4^{\circ}, 20.6^{\circ}\right)$ (thin), $\left(19.7^{\circ}, 60.9^{\circ}\right)$ (dashed).

Therefore we find the variations $\Delta \Phi$ of the angle of rotation $\Phi$ with respect to a uniform rotation by integrating (19):

$\Delta \Phi=\int \omega_{0} \times \frac{\mathrm{d} \omega}{\omega} \mathrm{d} t$

Where $\omega_{0}=2 \pi / T_{0}, T_{0}$ being the nominal value of the rotation period.

\subsection{Numerical results}

The value of the Love number $k_{2}$ of the asteroid necessary for our computations is not known. For the purpose we can refer to detailed theoretical discussions of the values of both static and dynamic Love numbers of asteroids in general and of binary asteroids in particular (Jacobson \& Scheeres 2011; Efroimsky 2015). Moreover, Goldreich \& Sari (2009) show that the Love number of asteroids scales with the asteroid's size, and is crucially dependent on whether the asteroid is a rubble pile or monolith. This uncertainty is likely more significant than the uncertainty in the density of the asteroid. For our computations of $\Delta \Phi$ we have set an arbitrary value of the Love number to $k_{2}=0.25$. Moreover we have adopted a ratio of density $\rho_{\oplus} / \rho_{\mathrm{A}}=2.3$ which corresponds to $\rho_{\mathrm{A}} \approx 2.4$. That estimation is quite in agreement with the generally accepted value for $S$ class asteroids (Krasinsky et al. 2002) to which Apophis belongs. Anyway the following results could be changed easily in a proportional way with respect to a new improved determination of the ratio $k_{2} / \rho_{\mathrm{A}}$ in the future.

Then we have considered three different pairs of initial conditions $\left(\lambda_{0}, \varepsilon_{0}\right)$, corresponding respectively to the nominal value $\left(\left(\lambda_{0}, \varepsilon_{0}=\left(36^{\circ}, 32^{\circ}\right)\right)\right.$, to the maximum variation of $\Delta \psi\left(\left(\lambda_{0}, \varepsilon_{0}\right)=\left(96.4^{\circ}, 20.6^{\circ}\right)\right)$ and to the maximum variation of $\Delta \varepsilon\left(\left(\lambda_{0}, \varepsilon_{0}\right)=\right.$ $\left.\left(55.0^{\circ}, 44.6^{\circ}\right)\right)$. Results are shown in Fig. 9 where we can remark that the amplitude of the angle $\Delta \Phi$ which represents the angular variation with respect to a uniform rotation, does not exceed $0.1^{\circ}$, which corresponds to a rotational time delay $\Delta T$ such that $\Delta T / T \approx 2 \times 10^{-4}$ and consequently $\Delta T \approx 30^{\mathrm{s}}$. We note that this amplitude is by two orders smaller than the amplitude of the variations of some values of $\Delta T$ deduced by Scheeres et al. (2005) from their Monte Carlo simulations.

\section{Discussion and future investigations}

In this paper we have calculated the variations of orientation of Apophis spin axis due to the Earth gravitational pull, during the close encounter between the two celestial bodies occuring on April 13 2029, with a minimum distance of roughly 6 Earth radii between the barycentres. For this purpose we have taken into account the considerable improvement concerning the determination of fundamental physical parameters. In particular we have used new accurate and well constrained values of the moments of inertia of the asteroid and a well restricted delimited zone of the position of the spin axis before the encounter. This precious information is taken from the recent study of Pravec et al. (2014) related to the spin state of Apophis.

Our computations encompassing the whole range of initial conditions show that, as it is the case for the orbital parameters the obliquity $\varepsilon$ and the longitude of the node $\psi$ of the equator (i.e. the equinox) of the asteroid could undergo dramatic changes (respectively $\Delta \varepsilon$ and $\Delta \psi$ ) during the close encounter, ranging respectively from $1.1^{\circ}$ to $6.1^{\circ}$ for $\Delta \varepsilon$ and from $7.1^{\circ}$ to $38.1^{\circ}$ for $\Delta \psi$. In particular we show with our computations that the influence of the triaxial component $H_{\mathrm{t}}=0.0887$ of the asteroid is at the same level as the influence of the dynamical ellipticity $H_{\mathrm{d}}=0.2125$, which is quite different for the planets for which it can be neglected in the computations at the first order of the precession-nutation.

Moreover we have calculated a rough estimation of the relative changes of the spin rate $\mathrm{d} \omega / \omega$ of Apophis and the corresponding variations $\Delta \Phi$ of the rotation angle $\Phi$ during the flyby, due to the zonal deformations of the asteroid under the gravitational perturbation exerted by the Earth. Our results show that the effect is relatively small in comparison with the effects related to the orientation of the spin axis. The measured absolute amplitude of $\Delta \psi$ does not exceed $30^{\mathrm{s}}$ for a rotation period $T=27.8 \mathrm{~h}$, which corresponds to an angular offset not exceeding $0.1^{\circ}$. A more complete study based on a 3D diagram involving all the possible initial conditions, although seemingly interesting, is beyond the scope of this paper. 
The effect of the fly-by on the polhody of Apophis is not investigated in this paper. This motion, as it is the case for the Earth, represents the relative position of the axis of rotation with respect to the figure axis, which can be identified to the axis with maximum moment of inertia. It can be characterized by two polar coordinates: the first, $\rho$, is the angle between the two axes (rotation and figure). The second, $\theta$, gives the direction of the segment joining the two axes with respect to a body-fixed reference frame. The polhody is named as "wobble" in (Pravec et al. 2014). After a careful examination of that paper it appears that the fact that Apophis be in a moderately excited SAM state with a ratio $E / E_{0}=1.024 \pm 0.013$ implies that the angle $\rho$ remains very small, whereas the angle $\theta$ undergoes quite substantial variations with an average amplitude of $\theta_{\text {aver. }}=37^{\circ}$ and extrema at $\theta_{\min .}=12^{\circ}$ and $\theta_{\max }=55^{\circ}$. The interactions between the variations in the angles $J, \rho$ and $\theta$ is complex and beyond the scope of the present study. However the problem deserves a deeper understanding and will be investigated in a future paper.

Another limitation of our study concerns our computations of the changes of orientation of the polar axis in space in Sect. 4 . This lies in the fact that for these computations we have admitted the hypothesis that during the fly-by the rotational angular rate $\omega$ of the asteroid remains constant. In fact that is certainly not true but the estimation of the amplitude of the variations of $\omega$ during the fly-by looks as an open question. In Sect. 5 we have shown that the changes of $\omega$ due to the sole tidal modification of the moment of inertia $C$ would be quite small, and maybe does not affect significantly our results. However a more complete study based on the important work of Scheeres et al. (2000) that investigates in a general analytical way the problem of the effects of gravitational interactions on asteroid spin rate would be necessary if we want to achieve a model more realistic than that proposed here. Yet as it was mentioned at the end of the last section a preliminary estimation of the changes in the effective rotation period of Apophis was done by Scheeres et al. (2005) just after the discovery of the asteroid. That study, based on Monte-Carlo simulations but without precise knowledge of the physical characteristics of the asteroid, shows that the rotation period could be subject in some cases to significant changes following the fly-by, but that in a lot of cases does not exhibit such important changes. In consequence, the influence of the variations of $\omega$ on the scaling factors $K$ and $K^{\prime}$ from Eq. (15) as well as of the calculations of $\Delta \psi$ and $\Delta \varepsilon$ in Eqs. (13) and (14) should be the purpose of a next study, without bringing into question their validity, but only their more rigorous application.

Notice that our computations could be also refined in the future by taking a more realistic model of the asteroid, assimilated here as a rigid body, in the case of the calculations of precession-nutation, or a simple elastic one, in the case of the calculations of variations of the rotation rate. In addition better constraints on initial conditions, in particular concerning the spin axis orientation just before the fly-by will surely be obtained from future observational campaigns. Finally, the propagation of the uncertainties of the moments of inertia of the asteroid on the results should deserve a peculiar complementary study. Nevertheless we estimate that our calculations provide interest for trying to detect the large amplitude of the variations of the spin axis orientation during the fly-by, for example by using radar antennas as Arecibo and Goldstone.

\section{References}

Bancelin, D., Colas, F., Thuillot, W., \& Assafin, M. 2012, A\&A, 544, A15

Bottke, W. F., Vokroulicky, D., Rubincam, D. P., \& Nesvorny, D. 2006, Ann. Rev. Earth Planet Sci., 34, 157

Bouquillon, S., \& Souchay, J. 1999, A\&A, 345, 282

Chesley, S. R., Milani, A., Tholen, D., et al. 2009, AAS Abstract Meeting, 41, 43.06

Cottereau, L., Souchay, J., \& Aljbaae, S. 2010, A\&A, 515, A9

Efroimsky, M. 2015, AJ, 150, 98

Farnocchia, D., Chesley, S. R., Chodas, P. W., et al. 2013, Icarus, 224, 192

Garradd, G. J. 2004, MPE Circ., 2004-Y25

Giorgini, J. D., Benner, L. A. M., Ostro, S. J., Nolan, M. C., \& Busch, M. W. 2008, Icarus, 193, 1

Goldreich, P., \& Sari, R. 2009, ApJ, 691, 54

Jacobson, S. A., \& Scheeres, D. J. 2011, ApJ, 736, L19

Kinoshita, H. 1977, Celest. Mech. Dyn. Astr., 15, 277

Krasinsky, G. A., Pitjeva, E. V., Vasilyev, M. V., \& Yagudina, E. I. 2002, Icarus, 158, $98 \mathrm{~K}$

Lhotka, C., Souchay, J., \& Shahsavari, A. 2013, A\&A, 556, A8

Melchior, P. 1983, The Tides of the Planet Earth, 2nd. Ed. (Oxford: Pergamon Press)

Petit, A., Souchay, J., \& Lhotka, C. 2014, A\&A, 565, A79

Pravec, P., Scheirich, P., Durech, J., et al. 2014, Icarus, 233, 48

Sansaturio, M. E., \& Arratia, O. 2008, Earth Moon and Planets, 102, 425

Scheeres, D. J., Ostro, S. J., Werner, R. A., Asphaug, E., \& Hudson, R. S. 2000, Icarus, 147, 106

Scheeres, D. J., Benner, L. A. M., Ostro, S. J., et al. 2005, Icarus, 178, 281

Smalley, K. E. 2004, MPE Circ., 2004- Y25

Souchay, J., Loysel, B. , Kinoshita, H., \& Folgueira, M. 1999, A\&AS, 135, 111

Souchay, J., Heron, G., Puente, V., \& Folgueira, M. 2014a, Rom. Astron. J., 24, 87

Souchay, J., Souami, D., Lhotka, C., Puente, V., \& Folgueira, M. 2014b, A\&A, 563, A24

Tucker, R., Tholen, D., \& Bernardi, F. 2004, MPS, 109613 